\title{
LETTER
}

\section{Non-abelian statistics in the interference noise of the Moore-Read quantum Hall state}

\author{
Eddy Ardonne ${ }^{1,2}$ and Eun-Ah Kim ${ }^{3}$ \\ ${ }^{1}$ Center for the Physics of Information, California Institute of Technology, \\ Pasadena, CA 91125, USA \\ 2 Microsoft Station Q, University of California, Santa Barbara, CA 93106, USA \\ 3 Stanford Institute for Theoretical Physics and Department of Physics, \\ Stanford University, Stanford, CA 94305, USA \\ E-mail: ardonne@nordita.org and eunahkim@stanford.edu
}

Received 14 February 2008

Accepted 10 March 2008

Published 8 April 2008

Online at stacks.iop.org/JSTAT/2008/L04001

doi:10.1088/1742-5468/2008/04/L04001

\begin{abstract}
We propose noise oscillation measurements in a double point contact, accessible with current technology, to seek for a signature of the nonabelian nature of the $\nu=5 / 2$ quantum Hall state. Calculating the voltage and temperature dependence of the current and noise oscillations, we predict the nonabelian nature to materialize through a multiplicity of the possible outcomes: two qualitatively different frequency dependences of the nonzero interference noise. Comparison between our predictions for the Moore-Read state with experiments on $\nu=5 / 2$ will serve as a much needed test for the nature of the $\nu=5 / 2$ quantum Hall state.
\end{abstract}

Keywords: fractional QHE (theory) 
Non-abelian quantum Hall (QH) states, such as the Moore-Read (MR) QH state [1], are considered to be the most promising route [2] to fault tolerant topological quantum computation [3]. The possibility of the $\nu=5 / 2 \mathrm{QH}$ plateau [4] being the MR state $[5,6]$ attracted interests from a wide range of fields: from string theory to solid state physics. A configuration of many non-abelian excitations, such as MR quasi-holes/particles (qhs/qps), is associated with a set of degenerate states. An exchange of two such excitations amounts to a rotation in the degenerate state space: the most exotic form of statistics allowed in two space dimensions. For the MR state, the $2 n$ qh state is $2^{n-1}$ fold degenerate [7] and four qhs can form a single quantum bit (qubit). This notion is at the heart of the current enthusiasm for the MR state, from both a fundamental science and an application oriented view. However, non-abelian statistics has not been observed to date.

There are proposals for detecting non-abelian statistics of the MR state by exploiting the braiding properties of underlying Chern-Simons theories [8]-[12]. Effects of nonabelian statistics on the non-linear transport of a single point contact have also been predicted $[13,14]$. While a signature of non-abelian statistics is yet to be observed, a recent experiment [15] demonstrated the feasibility of a $\nu=5 / 2$ single point contact (PC), whose qualitative tunneling characteristics are those of the MR edge state. Thus, the edge states can be used as probes [16] of the exotic topological order associated with the $\nu=5 / 2$ state

In this letter, we propose feasible noise measurements in a double PC interferometer and give a detailed prediction of clear, qualitative signatures of the non-abelian statistics at finite temperature and voltage. A noise spectrum is a powerful probe for the nature of excitations since it is determined by the dynamical properties containing information about the excited states. It is well known (see [17]) that the noise spectrum of an electronic system in an appropriate geometry can contain statistics-dependent features that are not contained in the dc conductance. It is natural to expect that the noise spectrum of strongly interacting systems, such as quantum Hall liquids, should exhibit an even richer behavior such as the one found in the case of the abelian fractional QH states ([18]), and for the non-abelian case in this paper. Here we focus on the double PC setup for two reasons. (i) It is the only interferometer that has been experimentally realized in (abelian) fractional QH states [19] which was subsequently analyzed theoretically [20]. (ii) By attaching leads to the edge states of this setup it is possible to realize the physical situation of the four-qh state which is the simplest state in which the consequences of their non-abelian statistics become directly observable. We examine the oscillatory part of the noise as a cross-current fluctuation and present the leading-order perturbation theory result. Our results apply to both abelian and non-abelian cases. In addition, we provide an interpretation of the 'even-odd effect' $[9,10]$ in the context of the edge state theory.

Double PC interferometer. The double PC setup was first proposed as a testbed for abelian fractional statistics [22], and there have been discussions on using the setup to detect non-abelian statistics [8]-[10], [23]. It was first pointed out in [23] that the interference between two different paths of adiabatic transport surrounding a region with localized qhs can be used to measure the associated non-abelian braiding. While this picture provides conceptual intuition, an explicit calculation in terms of the edge theory is still needed.

The edge state theory relevant for the low-energy dynamics of gapless edge excitations of the MR state consists of two parts: the standard free chiral boson $\varphi_{\mathrm{c}}$ describing the 
Non-abelian statistics in the interference noise of the Moore-Read quantum Hall state

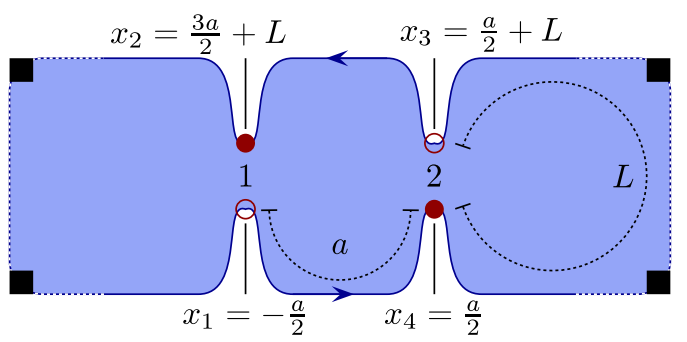

Figure 1. The double point contact setup, indicating the four positions $x_{i}$, $i=1, \ldots, 4$ associated with the point contacts 1 and 2 , in terms of the chiral abscissa coordinate $x$ (defined modulo $2 a+2 L$ ) for the chiral edge.

charge modes [24] with $\mathcal{L}_{\mathrm{c}}=1 /(2 \pi) \partial_{x} \varphi_{\mathrm{c}}\left(\partial_{t}+v \partial_{x}\right) \varphi_{\mathrm{c}}$, where $v$ is the edge mode velocity, and an additional charge neutral part: the chiral Ising conformal field theory (CFT), with a free Majorana field $\psi$ and the spin-field $\sigma$ [16]. The non-abelian nature of the Ising CFT is encoded in the fusion rule $\sigma \times \sigma=\mathbf{1}+\psi$, which makes the correlator of multiple $\sigma$ 's to form multi-dimensional conformal blocks [25]. The qh creation operator $\sigma \mathrm{e}^{\mathrm{i} / \sqrt{8} \varphi}(z)$ $(z \equiv \mathrm{i}(v t-x))$ is the most relevant operator in the renormalization group sense.

The manifestly relativistic nature of the edge CFT in $1+1$ dimensions reflects the general covariance (or topological invariance) of the underlying 2+1-dimensional Chern-Simons theory [26], as a low-energy effective field theory of the quantum Hall liquid $[23,27]$. An edge qh operator $\sigma \mathrm{e}^{\mathrm{i} \sqrt{8} \varphi}(z)$ 'marks' a point on the $1+1 \mathrm{D}$ surface, which corresponds to an end point of a Wilson line in the $2+1 \mathrm{D}$ Chern-Simons theory bounded by the surface (see figure 2(a)). Witten first showed that the multidimensionality of CFT correlators represented by the fusion rules reflects the nonabelian statistics of the corresponding qhs/qps represented by the associated Wilson lines. Moore and Read [1] proposed to interpret CFT correlators to represent manybody wavefunctions for quantum Hall states, now with the complex coordinate $z \equiv$ $x+\mathrm{i} y$ defined in $2+0$ dimensions (see figure $2(\mathrm{~b})$ ). The MR state wavefunction so constructed from the Ising CFT became a candidate description of the $\nu=5 / 2$ state [5]. Nayak and Wilczek [7] further demonstrated the non-abelian nature of the four-qh wavefunction through explicit exchange operations. While the wavefunction gives a clear physical picture of the nature of the state, it in itself is not a measurable quantity. On the other hand, the edge CFT can bridge between the theoretical structure and measurements.

We start by observing that a double PC allows one to access the four- $\sigma$ correlator in the $1+1$ D edge CFT (figure 2(a)). A quantum mechanical tunneling event annihilates a particle at one side of a point contact while creating one on the other side. Tunneling response naturally calls for contributions from four point functions with different ordering of $\sigma$ operators in this event space, at leading order. Hence, the tunneling response incorporates the effects of exchange in the $1+1 \mathrm{D}$ event space.

Perturbative calculation. We model the double PC setup with separation a between two PCs using a single abscissa coordinate $x$, defined modulo $2 a+2 L$ (see figure 1 ) and time $t$, which parameterize a cylinder. By taking the limit $L \rightarrow \infty$ at the end of the calculation, we take the effect of the leads into account properly without allowing the edge current to 


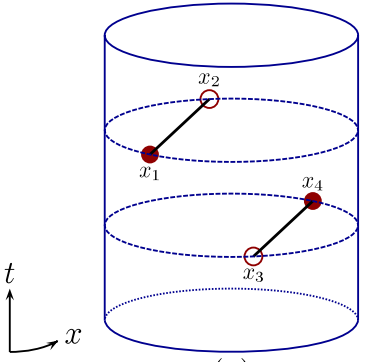

(a)

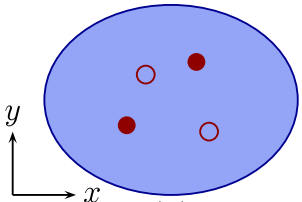

(b)

Figure 2. Four points marked in (a) $1+1 \mathrm{D}$ 'event' space and in (b) $2+0 \mathrm{D}$ space. CFT correlators associated with marked points are interpreted as (a) 'vacuum expectation values' associated with current carrying gapless edge modes (the black lines represent the Wilson lines, see text), (b) 'wavefunctions' associated with excitation configurations with finite energy.

go around the whole sample ${ }^{4}$. While it is typical to combine two chiral modes to form a single non-chiral mode $[14,22]$, it is not possible to take such approach for a MR multiPC setup without losing the information about its intricate topological structure. Our procedure allows us to describe the system using a single chiral edge mode; we checked it against the non-chiral mode approach in the abelian case [29].

The operators which tunnel a qp at PCs 1 and 2 are

$$
\begin{aligned}
& \hat{V}_{1}(t)=\sigma\left(x_{1}, t\right) \sigma\left(x_{2}, t\right) \mathrm{e}^{\mathrm{i} / \sqrt{8} \varphi_{\mathrm{c}}\left(x_{1}, t\right)} \mathrm{e}^{-\mathrm{i} / \sqrt{8} \varphi_{\mathrm{c}}\left(x_{2}, t\right)}, \\
& \hat{V}_{2}(t)=\sigma\left(x_{3}, t\right) \sigma\left(x_{4}, t\right) \mathrm{e}^{\mathrm{i} / \sqrt{8} \varphi_{\mathrm{c}}\left(x_{3}, t\right)} \mathrm{e}^{-\mathrm{i} / \sqrt{8} \varphi_{\mathrm{c}}\left(x_{4}, t\right)},
\end{aligned}
$$

which accounts for the creation and annihilation of qps on opposite edges at equal time (we note that $\sigma$ is self dual). The appropriate tunneling Hamiltonian and the current operator are then $\hat{H}_{\text {tun }}(t)=\sum_{j} \Gamma_{j}(t) \hat{V}_{j}(t)+$ h.c. and $\hat{I}(t)=\mathrm{ie}^{*} \sum_{j} \Gamma_{j}(t) \hat{V}_{j}(t)+$ h.c. [21]. Here the time-dependent tunneling strength is given by $\Gamma_{j}(t)=\Gamma_{j} \mathrm{e}^{\mathrm{i} \omega_{0} t}$, with $\omega_{0}=\frac{e^{*} V}{\hbar}$ the Josephson frequency and $e^{*}=e / 4$ the charge of the tunneling quasi-particle. In a magnetic field, the Aharonov-Bohm phase acquired by tunneling qps can be effectively incorporated through a flux-dependent relative phase between two tunneling amplitudes as $\Gamma_{1} \Gamma_{2}^{*}=\left|\Gamma_{1} \Gamma_{2}\right| \mathrm{e}^{\mathrm{i} \phi / \Phi_{0}}[22]$. We will assume that the tunneling is sufficiently weak at finite temperature and voltage and that the lowest Landau level is inert. We only consider tunneling of the most relevant quasi-holes.

We now calculate the average steady state current

$$
\langle\hat{I}\rangle=-\mathrm{i} \int_{-\infty}^{t} \mathrm{~d} t^{\prime}\left\langle\left[\hat{I}(t), \hat{H}_{\text {tun }}\left(t^{\prime}\right)\right]\right\rangle,
$$

which in general is a highly non-linear function of $V$ and $T$ for finite separation $a$, and the non-equilibrium noise

$$
S(\omega)=\frac{1}{2} \int_{-\infty}^{\infty} \mathrm{d} t^{\prime} \mathrm{e}^{\mathrm{i} \omega t^{\prime}}\left\langle\left\{\hat{I}(t), \hat{I}\left(t^{\prime}\right)\right\}\right\rangle,
$$

\footnotetext{
${ }^{4}$ This approach was carefully adopted in a different context in [28].
} 
which we define as the usual two point correlations involving the operator $\hat{I}[21]$. Notice that the current, which is a causal response, involves a commutator, while the noise, which is a fluctuation, involves an anti-commutator. This basic fact, when applied to $1+1 \mathrm{D}$ edge tunneling transport, has non-trivial consequences, both in that equations (2) and (3) require exchange in the event space and that one is restricted by causality while the other is not. It is amusing that both equations (2) and (3) explicitly depend on the four- $\sigma$ correlator at lowest order in $\Gamma$, which can take two possible values due to the non-abelian nature of the $\sigma$ operators. We label these two possibilities by $p=0,1$.

To leading order, the current and noise are $\left(e^{*}=1\right)$

$$
\begin{aligned}
\langle\hat{I}\rangle^{(p)} \equiv\langle\hat{I}\rangle_{d}^{(p)}+\cos \left(\frac{\phi}{\Phi_{0}}\right)\langle\hat{I}\rangle_{\mathrm{osc}}^{(p)} & \\
& =\Re \int_{0}^{\infty} \mathrm{d} t \sum_{j, k=1}^{2} \Gamma_{j} \Gamma_{k}^{*}\left[\mathrm{e}^{-\mathrm{i} \omega_{0} t}\left(\left\langle\hat{V}_{j} \hat{V}_{k}^{\dagger}\right\rangle^{(p)}(t)-\left\langle\hat{V}_{k}^{\dagger} \hat{V}_{j}\right\rangle^{(p)}(t)\right)\right] \Omega \\
\langle S(\omega)\rangle^{(p)} \equiv & \langle S(\omega)\rangle_{d}^{(p)}+\cos \left(\frac{\phi}{\Phi_{0}}\right)\langle S(\omega)\rangle_{\mathrm{osc}}^{(p)} \\
& =\Re \int_{-\infty}^{\infty} \mathrm{d} t \sum_{j, k=1}^{2} \Gamma_{j} \Gamma_{k}^{*}\left[\mathrm{e}^{\mathrm{i}\left(\omega-\omega_{0}\right) t}\left(\left\langle\hat{V}_{j} \hat{V}_{k}^{\dagger}\right\rangle^{(p)}(t)+\left\langle\hat{V}_{k}^{\dagger} \hat{V}_{j}\right\rangle^{(p)}(t)\right)\right]
\end{aligned}
$$

with the Aharonov-Bohm oscillatory parts which require coherence between two PCs, and the direct parts which only involve a single PC. The correlators $\left\langle\hat{V}_{j} \hat{V}_{k}^{\dagger}\right\rangle^{(p)}(t)^{\text {'s can }}$ be calculated at finite temperature $T$ (in units where $k_{\mathrm{B}}=1$ and $v=1$ ) in terms of $s(x, t) \equiv \mathrm{i} \sinh (\pi T(x+t))$ using the standard conformal mapping:

$$
\begin{aligned}
\left\langle\hat{V}_{1} \hat{V}_{2}^{\dagger}\right\rangle^{(p)}(t)= & \sqrt{\pi T / 2} \frac{(-1)^{p} s(a+L, t)^{1 / 4} s(-a-L, t)^{1 / 4}}{s(-L, t)^{1 / 4} s(-a-2 L, t)^{1 / 4} s(a, t)^{1 / 4} s(-a, t)^{1 / 4}} \\
& \times \sqrt{1+(-1)^{p} \sqrt{\frac{s(a, t) s(-a, t)}{s(a+L, t) s(-a-L, t)}}} .
\end{aligned}
$$

Here, the overall phase $(-1)^{p}$ is determined by requiring that the current flows in the direction of the applied bias voltage. We used the chiral boson correlator combined with the four- $\sigma$ correlator

$$
\begin{aligned}
\left\langle\sigma\left(z_{1}\right) \sigma\left(z_{2}\right)\right. & \left.\sigma\left(z_{3}\right) \sigma\left(z_{4}\right)\right\rangle^{(p)} \\
& =\frac{1}{\sqrt{2}}\left(z_{1}-z_{2}\right)^{-1 / 8}\left(z_{3}-z_{4}\right)^{-1 / 8}(1-\xi)^{-1 / 8} \sqrt{1+(-1)^{p} \sqrt{1-\xi}},
\end{aligned}
$$

with the cross-ratio $\xi=\left(z_{1}-z_{2}\right)\left(z_{3}-z_{4}\right) /\left(z_{1}-z_{4}\right)\left(z_{3}-z_{2}\right)$ [25]. Note that this expression is valid for $\left|z_{1}\right|<\left|z_{2}\right|<\left|z_{3}\right|<\left|z_{4}\right|$. For other orderings, one has to analytically continue the expression above.

Hearing the non-abelian statistics. Rather unexpectedly, we find that the channel dependence, a hallmark of non-abelian statistics, shows up in the noise but not in the current. This is due to an intriguing interplay between the inherently relativistic nature of the edge state theory and the causal nature of current as a response. The evaluation of the tunneling current and the noise of equations (4) and (5) requires combining four 

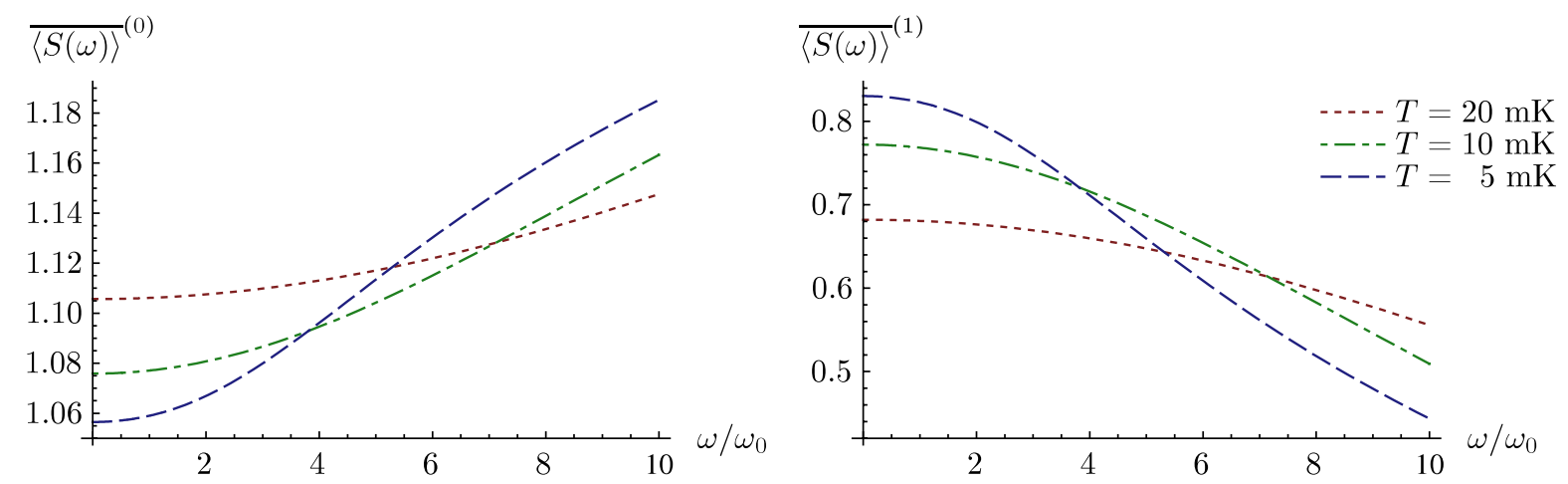

Figure 3. The (dimensionless) amplitude of the noise oscillations $\overline{\langle S(\omega)\rangle}^{(p)}$ in two states (0) (left) and (1) (right) for $a=5 \mu \mathrm{m}, V=.38 \mu \mathrm{V}\left(\omega_{0}=100 \mathrm{MHz}\right)$, $v=1.10^{7} \mathrm{~m} \mathrm{~s}^{-1}$, for various temperatures.

different terms of the type $\left\langle\hat{V}_{j} \hat{V}_{k}^{\dagger}\right\rangle^{(p)}(t)$. Explicitly exchanging these operators by means of analytic continuation, with much attention to branch cuts and taking the limit $L \rightarrow \infty$ afterward, we find for the Aharonov-Bohm oscillation amplitude of the current and the noise [29]

$$
\begin{gathered}
\langle\hat{I}\rangle_{\mathrm{osc}}^{(p)}=4 e^{*} \sqrt{\pi T}\left|\Gamma_{1} \Gamma_{2}\right| \int_{a}^{\infty} \mathrm{d} t \frac{\sin \left(\omega_{0} t\right)}{\sinh (\pi T(t-a))^{1 / 4} \sinh (\pi T(t+a))^{1 / 4}} \\
\langle S(\omega)\rangle_{\mathrm{osc}}^{(p)}=4\left(\mathrm{e}^{*}\right)^{2} \sqrt{\pi T}\left|\Gamma_{1} \Gamma_{2}\right| \times\left(\int_{a}^{\infty} \mathrm{d} t \frac{\cos \left(\left(\omega+\omega_{0}\right) t\right)+\cos \left(\left(\omega-\omega_{0}\right) t\right)}{\sinh (\pi T(t-a))^{1 / 4} \sinh (\pi T(t+a))^{1 / 4}}\right. \\
\left.+(-1)^{p} \int_{0}^{a} \mathrm{~d} t \frac{\sqrt{2}\left(\cos \left(\left(\omega+\omega_{0}\right) t\right)+\cos \left(\left(\omega-\omega_{0}\right) t\right)\right)}{\sinh (\pi T(t-a))^{1 / 4} \sinh (\pi T(t+a))^{1 / 4}}\right)
\end{gathered}
$$

The direct, single PC contributions which yields the shot noise results $\left(S(0)=e^{*}\langle I\rangle\right)$ can be obtained from equations (8) and (9) by taking the limit $a \rightarrow 0$. The above results can be generalized to other fractional QH states in a straightforward manner. Note that for (abelian) Laughlin states at filling $\nu=1 / m$, the exponents change from $1 / 4$ to $1 / m$, and only the $p=0$ state is possible.

The state $(p)$ dependence only appears in the second term of the noise oscillation equation (5), which is absent in the single PC limit. In order to understand this we note that the 'light cone' $|t|=a$ (here the speed of light is the edge mode speed $v \equiv 1$ ) divides the causally connected (time-like separated) region $|t|>a$ from the space-like separated region $|t|<a$, for the correlator equation (6). Due to the branch cut structure, the correlators behave differently under exchange of operators in these two event space regions, and we find the channel dependence to vanish in any causally connected regions $(|t|>a)$ [29]. Hence the current, which is a causal response (see equation (2)), is state independent. In contrast, the noise, which is a fluctuation unrestricted by causality (see equation (3)), will display state dependence when the space-like separated contribution is significant.

Figure 3 shows a clear, qualitative difference in the frequency dependence of the noise oscillations in the two states: $p=0$ and $p=1$. Here we plotted the dimensionless noise oscillation amplitude $\overline{\langle S(\omega)\rangle}^{(p)} \equiv\langle S(\omega)\rangle_{\text {osc }}^{(p)} /\langle S(\omega)\rangle_{d}$ (assuming $\left|\Gamma_{1}\right|=\left|\Gamma_{2}\right|$ ) for different temperatures and parameters within reach of current technology. We find that there is an 
Non-abelian statistics in the interference noise of the Moore-Read quantum Hall state

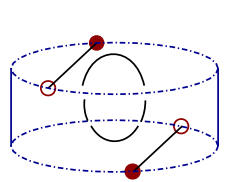

(0)

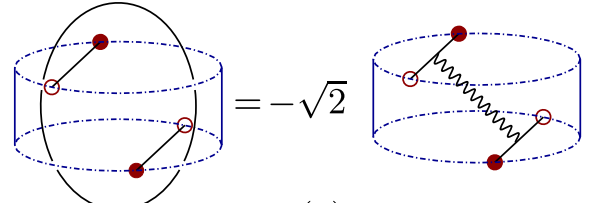

(1)

Figure 4. Two distinct states of double PC. Left: a Wilson loop which can be shrunk to a point. Right: a Wilson loop looping around both Wilson lines, which is equivalent to two Wilson lines exchanging a $\psi$ up to a factor of $-\sqrt{2}$.

optimal range for the distance $a$. If $a$ is too small, the system reaches the single PC limit without state dependence. On the other hand, if $a$ becomes comparable to the thermal decoherence scale set by $T$, interference features get washed out as $\exp (-a T)$ [29]. The qualitative difference in the two states traces back to the fact that the contributions from two event space regions are added for state (0) while they are to be subtracted for state (1) (see equation (9)). This relative negative sign for state (1) reflects the hidden Majorana fermionic character of this state which is symbolically represented in the second term of the fusion rule, $\sigma \times \sigma=\mathbf{1}+\psi$. Only in state (1) does the rearrangement of $\sigma$ 's needed in equations (4) and (5) effectively exchange two Majorana fermions. This fermionic nature results in the decreasing concave frequency dependence.

Effect of localized qhs. The distinction between these two equally allowed states is the configuration of bulk quasi-holes. We have depicted two topologically distinct Wilson line configurations corresponding to states (0) and (1) in figure 4. The underlying braid properties of the Wilson lines [29,30] for the two configurations of figure 4 allow the interference noise to access the two state nature (and hence the essence of the non-abelian statistics) of the MR state. The observed result depends on the state of the system. For instance, if the bulk quasiparticles are in a coherent linear superposition, the measured noise will be a linear combination of the results for states (0) and (1). This is in contrast to the abelian case which can only be in the pure state (0) since in this case the state is unique [29]. Hence, even all possible superpositions will have a signature that distinguishes the non-abelian state with two-dimensional Hilbert space from an abelian state.

The Aharonov-Bohm oscillations vanish for both the noise and the current when the Wilson line of a $\mathrm{qp}-\mathrm{qh}$ pair in the bulk loops around only one of the Wilson lines associated with the tunneling. In this case, one pair of $\sigma$ 's fuses to $\psi$ while the other fuses to $\mathbf{1}$, and thus the correlator equation (7) vanishes [29]. This is the edge-theory interpretation of the even-odd effect $[9,10]$. We have shown that there are two distinct possibilities, which we called (0) and (1), within the non-vanishing 'even' case which is evident in the interference noise. This provides an alternative way of looking for the signature of non-abelian statistics, which can easily be generalized for other non-abelian states.

Conclusion. We perturbatively calculated the tunneling current and noise of a double PC interferometer in the MR quantum Hall state using the associated edge state theory. This setup provides direct experimental access to the four- $\sigma$ correlator which describes two topologically distinct states. Exploiting the fact that the measurable quantities naturally involve exchange in the event space, we find that the Aharonov-Bohm oscillatory noise 
can be used to 'hear' a clear signature of non-abelian statistics. We predict a qualitative difference in the low-frequency behavior of the oscillatory noise between the two states. Our detailed predictions for the voltage and temperature dependence can be compared with future measurements. Due to the non-local entanglement between bulk and edge qhs, which is tied to the non-abelian nature, the preparation of a system in a pure state of any of the topologically distinct possibilities considered here or in $[9,10]$ requires the control of pinned bulk qhs. The problem of how to effectively control the state is an important and open question of direct relevance to experiments on non-abelian interferometers.

We thank P Bonderson, C Chamon, S B Chung, L Fidkowski, E Fradkin, M Freedman, C Nayak, S Shenkar, J Slingerland, K Shtengel, Z Wang for illuminating discussions. EAK was supported by the Stanford Institute for Theoretical Physics and in part by the Microsoft Station Q.

\section{References}

[1] Moore G and Read N, 1991 Nucl. Phys. B 360362

[2] Das Sarma S, Freedman M and Nayak C, 2006 Phys. Today 5932

[3] Kitaev A, 2003 Ann. Phys. 3032

[4] Willet R et al, 1987 Phys. Rev. Lett. 591776

[5] Greiter M, Wen X-G and Wilczek F, 1992 Nucl. Phys. B 374567

[6] Morf R H, 1998 Phys. Rev. Lett. 801505 Rezayi E H and Haldane F D M, 2000 Phys. Rev. Lett. 844685

[7] Nayak C and Wilczek F, 1996 Nucl. Phys. B 479529

[8] Das Sarma S, Freedman M and Nayak C, 2005 Phys. Rev. Lett. 94166802

[9] Stern A and Halperin B I, 2006 Phys. Rev. Lett. 96016802

[10] Bonderson P, Kitaev A and Shtengel K, 2006 Phys. Rev. Lett. 96016803

[11] Feldman D and Kitaev A, 2006 Phys. Rev. Lett. 97186803

[12] Hou C-Y and Chamon C, 2006 Phys. Rev. Lett. 97146802

[13] Bena C and Nayak C, 2006 Phys. Rev. B 73155335

[14] Fendley P, Fisher M P A and Nayak C, 2006 Phys. Rev. Lett. 97036801

[15] Miller J B et al, 2007 Nat. Phys. 3561

[16] Wen X-G, 1995 Adv. Phys. 44405

[17] Lesovik G B and Levitov L S, 1994 Phys. Rev. Lett. 72538

[18] Kim E-A et al, 2005 Phys. Rev. Lett. 95176402

[19] Camino F E, Zhou W and Goldman V J, 2005 Phys. Rev. Lett. 72155313

[20] Kim E-A, 2006 Phys. Rev. Lett. 97216404

[21] Chamon C, Freed D E and Wen X G, 1995 Phys. Rev. B 512363

[22] Chamon C et al, 1997 Phys. Rev. B 552331

[23] Fradkin E et al, 1998 Nucl. Phys. B 516704

[24] Wen X G, 1990 Phys. Rev. B 4112838

[25] Belavin A A, Polyakov A M and Zamolodchikov A B, 1984 Nucl. Phys. B 241333

[26] Witten E, 1989 Commun. Math. Phys. 121351

[27] Fradkin E, Nayak C and Schoutens K, 1999 Nucl. Phys. B 546711

[28] Kim E-A et al, 2006 Phys. Rev. B 74155324

[29] Ardonne E and Kim E-A, 2008 in preparation

[30] Moore G and Seiberg N, 1989 Commun. Math. Phys. 123177 